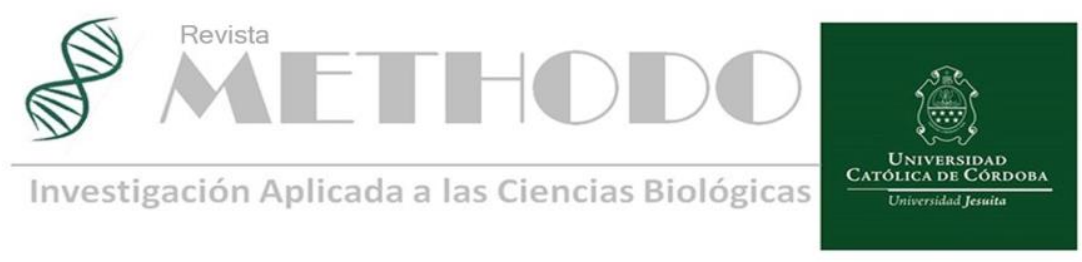

CASO CLINICO Rev. Methodo 2020;5(2):73-77

https://doi.org/10.22529/me.2020.5(2)07

Recibido 28 Nov. 2019 | Aceptado 27 Feb. 2020 |Publicado 20 Abr. 2020

\title{
Liquen plano oral. Reporte de un caso y revisión de la literatura
}

\section{Oral lichen planus. Case report and literature review}

\author{
Nicolás Leonardi ${ }^{1}$, Ricardo Caciva ${ }^{1}$, Eduardo D. Piemonte ${ }^{1}$, Martín Brusa $^{1}$, Carolina Gonzalez ${ }^{2}$, María \\ Eloisa Garay ${ }^{2}$, René Luis Panico ${ }^{1}$ \\ 1. Universidad Católica de Córdoba. Facultad de Ciencias de la Salud Medicina Bucal, Escuela de Odontología. \\ 2. Universidad Católica de Córdoba. Facultad de Ciencias de la Salud. Histología General y Dental, Escuela de Odontología. \\ Correspondencia: Nicolás Leonardi. email: nico_leonardi@hotmail.com.ar.
}

\section{Resumen}

El liquen plano oral (LPO) es una enfermedad mucocutánea crónica, de carácter inflamatorio, etiología desconocida y naturaleza autoinmune, en la que se produce una agresión por parte de los linfocitos $\mathrm{T}$ dirigida frente a las células basales del epitelio de la mucosa oral. El diagnóstico del LPO debe basarse en la observación clínica y confirmarse con la descripción de las características histopatológicas. Se presenta caso clínico de paciente femenina de 39 años de edad que acudió a consulta odontológica al Servicio de Estomatología de la Escuela de Odontología, de la Facultad de Ciencias de la Salud, Universidad Católica de Córdoba, por presentar múltiples lesiones blancas en la cavidad bucal de 1 año de evolución. La paciente manifiesta episodios de stress. Se realizó biopsia de tipo incisional y se envió a anatomía patológica donde se confirmó el diagnóstico de Liquen Plano Oral.

Palabras clave: Liquen Plano Oral, Mucosa Oral, Inflamación Crónica.

\begin{abstract}
Oral lichen planus (LPO) is a chronic mucocutaneous disease, of an inflammatory nature, unknown etiology and autoimmune nature, in which aggression by $\mathrm{T}$ lymphocytes directed against the basal cells of the oral mucosa epithelium occurs. The diagnosis of LPO should be based on clinical observation and confirmed with the description of histopathological characteristics. There is a clinical case of a 39-year-old female patient who attended a dental office at the Stomatology Service of the School of Dentistry, Faculty of Health Sciences, Catholic University of Córdoba for presenting multiple white lesions in the oral cavity of 1 year of evolution. The patient manifests episodes of stress. An incisional type biopsy was performed and sent to a pathological anatomy where the diagnosis of Oral Lichen Planus was confirmed.
\end{abstract}

Keywords: Lichen Planus Oral, Oral Mucosa, Chronic Inflammation.

\section{Introducción}

El liquen plano oral (LPO) es una enfermedad inflamatoria crónica mucocutánea de etiología desconocida y naturaleza autoinmune, en la que se produce una agresión $\mathrm{T}$ linfocitaria dirigida frente a las células basales del epitelio de la mucosa oral, afectando fundamentalmente a la piel, mucosa oral y genital, cuero cabelludo y uñas, siendo frecuente que las lesiones bucales precedan a las cutáneas o 
que sea única la localización intraoral ${ }^{1-3}$. Se ha estimado una prevalencia en la población general entre $0,5 \%$ a $3 \%$; con una proporción de hombres y mujeres de 1:2,5. El LPO puede presentarse en cualquier etapa de la vida, siendo más frecuente en mujeres entre la cuarta y séptima década ${ }^{4}$. La importancia de su conocimiento radica en que el LPO es capaz de malignizar, siendo considerado como un trastorno o desorden potencialmente maligno. Las formas clínicas de presentación de esta entidad han sido estudiadas en la bibliografía, destacando las variedades papular, reticular, atrófica, ampollar, erosiva y en placa ${ }^{5}$.

La variante reticular es la más frecuente apareciendo como una lesión blanca, lineal en forma estrellada, reticular o anular ligeramente sobreelevada, palpable denominadas estrías de Wickham y que no se desprenden al raspado. Se presenta generalmente en zonas de la mucosa yugal de manera bilateral, simétrica y asintomática ${ }^{6-7}$.

La variante atrófica-erosiva se caracteriza por un adelgazamiento del espesor del epitelio, manifestándose por un enrojecimiento generalizado y difuso exponiéndose los vasos sanguíneos subyacentes del corion. La localización más frecuente es la lengua, siendo también la encía un lugar habitual de asentamiento presentándose como una gingivitis descamativa crónica. Esta forma se caracteriza por la presencia de ulceraciones del epitelio sobre una mucosa atrófica, aunque también podría presentarse de forma ampollar ${ }^{8}$.

No se ve diferencia en el mecanismo patogénico entre el LPO reticular y el atrófico-erosivo, aunque histológicamente sí ya que se observa a nivel del corion diferencia en la densidad del infiltrado inflamatorio siendo mayor en los atrófico erosivos que en los reticulares ${ }^{9-10}$.

El diagnóstico del LPO debe basarse en la observación clínica y confirmarse con la descripción de las características histopatológicas. Hay una serie de criterios clínicos e histopatológicos importantes para confirmar el diagnóstico de una manera concluyente ${ }^{11}$. El patrón clínico característico es la presencia de lesiones bilaterales más o menos simétricas y de líneas blanquecinas en el patrón reticular o lesiones atróficas en la variante atrófico-erosivo. Los criterios histopatológicos son la presencia de una banda bien definida de infiltrado inflamatorio compuesta principalmente por linfocitos, la degeneración hidrópica de la capa basal y la ausencia de displasia. Una hiperqueratosis, que se define como un aumento de espesor de la capa córnea, una acantosis o aumento del número de capas de células granulares donde las papilas se disponen a modo de dientes de sierra. La lesión más significativa del LPO es la degeneración hidrópica del estrato basal que es la degeneración de los queratinocitos basales y está relacionada con la patogenicidad de la enfermedad. Aparecen los cuerpos de Civatte que son estructuras eosinófílas, redondeadas o lobuladas que a veces contienen núcleos picnóticos o fragmentos nucleares localizados principalmente en la capa basal. Serían los queratinocitos basales que han sufrido necrosis prematura por la degeneración hidrópica de las células basales ${ }^{12-13-14-15}$.

En la inspección clínica hay que ver su localización, siendo la más frecuente dentro de la cavidad bucal la mucosa yugal, lengua y encía, aunque también puede encontrarse en la mucosa labial, piso de boca, comisura labial y paladar. Al explorar las manifestaciones cutáneas se deben observar las áreas flexoras y dentro de ellas la cara anterior de las muñecas y otras regiones como la conjuntiva ocular, nariz y laringe ${ }^{16}$. La zona a biopsiar debe ser un área no ulcerada donde se conserve el epitelio y de ser posible que existan estrías $^{17}$

Respecto a los factores psicológicos en los que la mayoría de los autores están de acuerdo ya que juegan un importante papel en la evolución del LPO, se ha relacionado el estado de ánimo con la situación inmunológica y los niveles de cortisol especulándose sobre la posibilidad de que una alteración en el estado de ánimo pueda producir un impacto sobre el sistema neuroendocrino, que influiría sobre las subpoblaciones linfocitarias de los pacientes con LPO. Esta relación se encuentra más desarrollada en la variante atrófica-erosiva que en la reticular. Los pacientes con LPO tienen un mayor índice de ansiedad, sobre todo en las formas más agresivas de LPO atrófico-erosivo. Por lo tanto, además de ser un factor de riesgo la ansiedad también agravaría la lesión ${ }^{18-19-20}$.

\section{Caso clínico}

Paciente femenina de 39 años de edad que acudió a consulta odontológica al Servicio de Estomatología de la Escuela de Odontología, de la Facultad de Ciencias de la Salud, Universidad Católica de Córdoba por presentar múltiples lesiones blancas en la cavidad bucal de 1 año de evolución, con sintomatología de ardor ante comidas picantes e ingesta de cítricos y stress. Se automedico con un antimicótico local, nistatina suspensión oral $100.000 \mathrm{UI} / \mathrm{ml}$ durante 15 días, sin evolución favorable ni cambios clínicos, por lo cual decidió consultar a un especialista en Estomatología.

En los antecedentes personales patológicos los datos de relevancia son hipotiroidismo, la cual esta medicada con Levotiroxina 150mg. Respecto a los hábitos paciente fumadora de cigarrillos rubios en 
una cantidad de 5 por día desde los 16 años dando como resultado 41.975 cigarrillos fumados en la actualidad en 23 años, es bebedora social, consume cerveza rubia y toma mate a temperatura caliente 2lt diarios.

Al examen clínico podemos observar múltiples lesiones blancas distribuidas en la cavidad bucal afectando dorso lingual, ambas mucosas yugales, mucosa labial inferior y encía con zonas erosivas y áreas queratoticas con un dibujo característico en forma anular, lineales, reticulares con un ligero relieve y palpables que no se desprenden al raspado (Figura.1, 2 y 3).

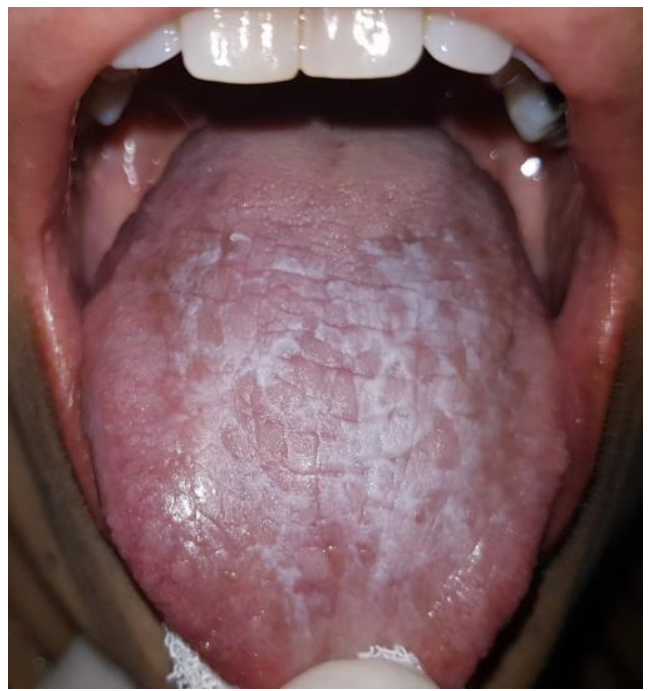

Figura 1. Lesiones blancas compatibles con mancha y queratosis afectando el dorso lingual.

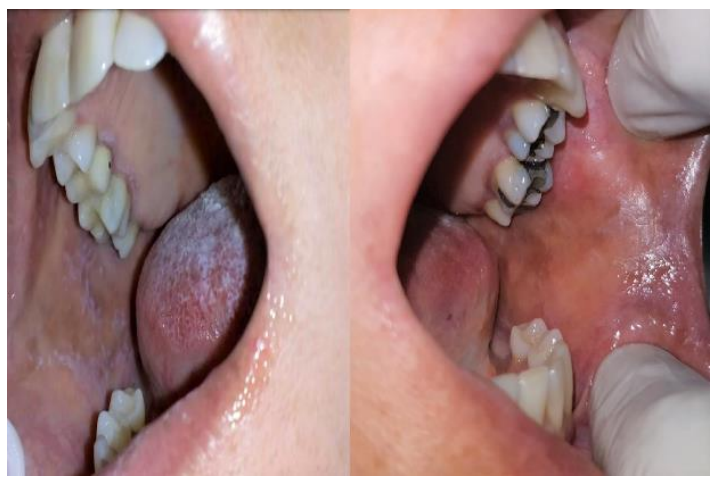

Figura 2. Epígrafe: Estrías de Wickam características de Liquen Plano en mucosa yugal.

Se solicitan estudios complementarios de diagnóstico, tales como análisis de rutina, se plantea posible diagnostico presuntivo y sus respectivos diagnósticos diferenciales.

Tras la evaluación clínica y los exámenes complementarios de diagnóstico, se realizaron dos biopsias de tipo incisional del sitio más representativo de la lesión, una de dorso lingual y otra de un área de mucosa yugal derecha, tercio medio, se las fijo en formol al $10 \%$ y se hicieron los estudios anatomopatológicos correspondientes para la confirmación diagnostica (Figura. 4).

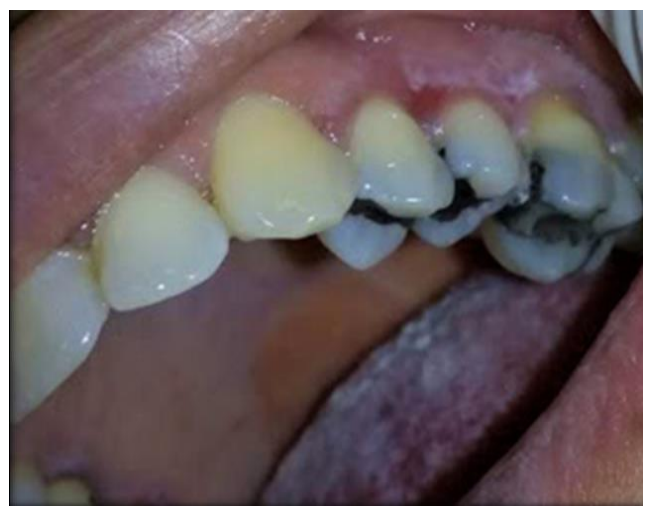

Figura 3. Lesión blanca en encía superior acompañada de una zona erosiva a nivel de la papila interdentaria del ED 24 Y 25.

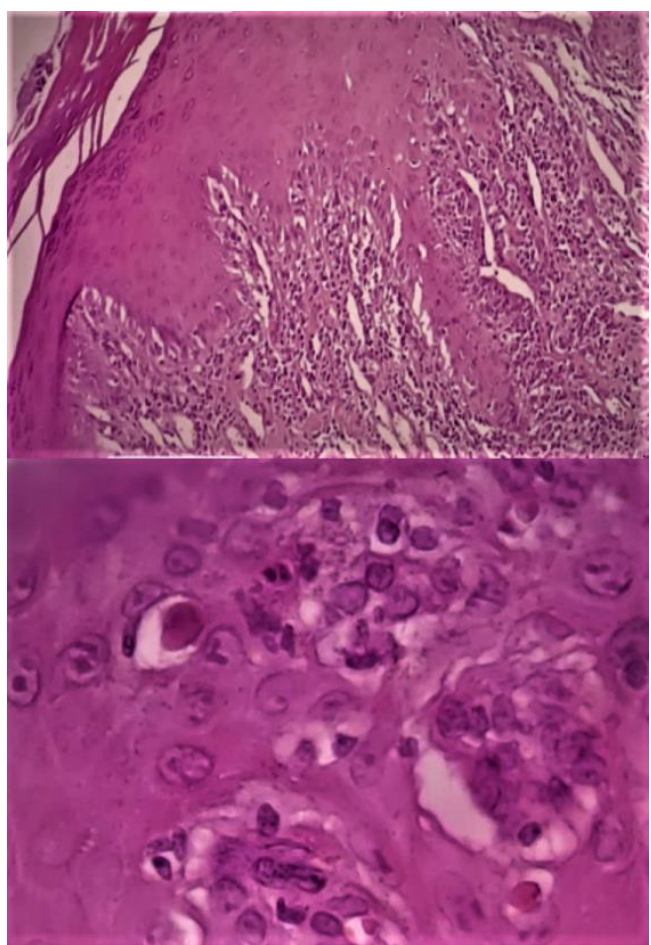

Figura 4. superior se puede observar acantosis y redes de crestas "en dientes de sierra". hiperqueratosis, hipergranulosis focal. Infiltrado inflamatorio linfocítico "en banda" con signos de degeneración hidrópica o licuefacción de la capa basal.

inferior se puede observar Cuerpos de "Civatte" también llamados cuerpos coloides, cuerpos hialinos o cuerpos citoides, corresponden a queratinocitos basales con necrosis aislada y prematura.

\section{Discusión}

En las enfermedades autoinmunes es importante establecer el diagnóstico definitivo, oportuno y certero, así como la correlación clínicohistopatológica; de esta manera se puede optar por protocolos terapéuticos con resultados aceptables.

Revista Methodo: Investigación Aplicada a las Ciencias Biológicas. Universidad Católica de Córdoba. Jacinto Ríos 571 Bo Gral. Paz. X5004FXS. Córdoba. Argentina. Tel.: (54) 3514517299 / Correo: methodo@ucc.edu.ar / Web: methodo.ucc.edu.ar | CASO CLINICO Rev. Methodo 2020;5(2):73-77 
El seguimiento clínico de los pacientes se debe realizar a largo plazo, para lograr la remisión de las lesiones, control de la enfermedad y evitar futuras complicaciones. Es fundamental el manejo interdisciplinario de los profesionales de la salud en los pacientes que sufren esta patología.

Aún existe controversia respecto al potencial de transformación del liquen plano. Se sabe que las células atacadas por los linfocitos $\mathrm{T}$ del mismo paciente desarrollan un complejo mecanismo molecular enfocado a detener el ciclo celular de la reparación de ADN o a inducir apoptosis con el fin de eliminar células dañadas en su estructura; algunos autores mencionan que estas células frecuentemente responden a este ataque con aumento en las tasas de proliferación, sin embargo, se requiere mayor evidencia científica que correlacione unidireccionalmente la etiología y el potencial oncogénico de la enfermedad ${ }^{21,22}$.

\section{Bibliografía}

1. Dekker Np, Lozada-Nür F, Lagenaur LA, MacPhail LA, Blom CY, Regezi JA. Apoptosis-associated markers in oral lichen planus. J Oral Pathol Med 1997; 26:170-5.

2. Neppelberg E, Johannessen Ac, Jonsson R. Apoptosis in oral lichen planus. Eur J Oral 2001; 109:361-4.

3. Bascones-Ilundain C, González-Moles MA, Carrillo-De Albornoz A, Bascones-Martínez A. Liquen plano oral (I). Aspectos clínicos, etiopatogénicos y epidemiológicos. 2006; 22(1):11-19.

4. Le Cleach L, Chosidow O. Lichen planus. N Engl J Med. 2012; 366:723-32.

5. López-López J, Omãna-Cepeda C, JanéSalas E. Oral precancer and cancer. MedClin (Barc). 2015; 145:404-8.

6. González Moles MA, Rodriguez-Arcilla A, Ruiz - Ávila I, Morales Garcia P. Liquen plano oral. RCOE 2000; 5:255-69.

7. Valeron F, Caballero M, Caballero R. Liquen plano oral. Espejo clínico 1999; 0:6-11.

8. Cerero-Lapiedra R, García-Núñez JA, GarcíaPola MJ. Liquen plano oral. RCOE 1997; 2:643-60.

9. Carozo M, Broccoletti R, Carbone M, Gandolfo S, Garzino P, Gascio G. Phenotipic análisis of peripheral blood cell immunity in Italian patients with varieties of oral lichen planus Bull Gropu Int Reach Sci Stomatol et Odontol 1996; 39:33-8
10. García-Pola MJ, Anitua MJ; Fernandez BE, García JM, López A. Estudio comparativo de la expresión del Ki-67 en el liquen plano oral y leucoplasia oral. Análisis cuantitativo. Medicina oral 2001; 6:364-7

11. Van der Meij E, Van der Waal I. Lack of clinicopathologic correlation in the diagnosis of oral lichen planus based on the presently available diagnostic criteria and suggestions for modifications. J Oral Pathol Med 2003; 32:507-12.

12. Neville BW, Dam DD, Allen CM, Bouquot JE. Oral \& maxillofacial pathology. EE. UU.: Saunders Elsevier; 2009.

13. Bascones A, Seoane JM, Aguado A, SuárezQuintanilla JM. Cáncer y precáncer oral. Bases clínicoquirúrgicas y moleculares. España: Ediciones Avances; 2003.

14. Hirota M, Ito T, Okudela $\mathrm{K}$, Kawabe R, Yazawa T, Hayashi H, et al. Cell proliferation activity and expression of cell cycle regulatory proteins in oral lichen planus. J Oral Pathol Med. 2002;31(4):204- 212. DOI: 10.1034/j.1600-0714.2002.310403.

15. White FH, Jin Y, Yang L. Quantitative cellular and nuclear volumetric alterations in epithelium from lichen planus lesions of human buccal mucosa. J Oral Pathol Med. 1994;23(5):205-208. DOI: 10.1111/j.16000714. 1994.tb01114.

16. Bagan JV, Cerero R. Liquen plano oral. En. Ceballos A. Bermejo A, Aguirre JM, Peñarocha M. Medicina oral. Barcelona: Masson 1995:202-19.

17. Blanco A, Gándara JM, Rodríguez A, García A, Rodríguez I. alteraciones bioquímicas y su correlación clínica con el liquen plano oral. Medicina Oral 2000; 5:14-25

18. García-Pola MJ y Cerero-Lapiedra R. Liquen plano en la mucosa oral. Revista clínica española 1998; vol. 1998,7:448-57.

19. Rödstrom PO, Jontell M, Hakeberg $M$, Berggren U, Lindstedt G. Erosive oral lichen planus and salivary cortisol. J Oral Pathol Med 2001;30: 257-63.

20. García-Pola MJ y Huerta G. Valoración de la ansiedad como factor etiológico del liquen plano oral. Medicina oral 2000; vol. 5,1:7-13.

21. Bloor BK, Malik FR, Odell EW, Morgan PR. Quantitative assessment of apoptosis in oral lichen planus. Oral Surg Oral Med Oral Pathol Radiol Endod. 1999;88(2):187-195. 
Leonardi N, Caciva R, Piemonte ED, Brusa M, González C, Garay ME, Pánico RL. Liquen plano oral. Reporte de un caso y revisión de la literatura.

22. Tobón-Arroyaye SI, Villegas-Acosta FA, Ruiz-Restrepo SM, Vieco-Durán M, Restrepo-Misas M, Londoño-López ML. Expression of caspase-3 and structural changes associated with apoptotic cell death of keratincytes in oral lichen planus. Oral Dis. 2004;10(3):173-178.

$$
\begin{aligned}
& \text { (c) (1)(2)(0) } \\
& \text { BY NC SA }
\end{aligned}
$$

